TRANSACTIONS OF THE

AMERICAN MATHEMATICAL SOCIETY

Volume 358, Number 4, Pages 1435-1454

S 0002-9947(05)04105-X

Article electronically published on November 18, 2005

\title{
SHARP DIMENSION ESTIMATES OF HOLOMORPHIC FUNCTIONS AND RIGIDITY
}

\author{
BING-LONG CHEN, XIAO-YONG FU, LE YIN, AND XI-PING ZHU
}

\begin{abstract}
Let $M^{n}$ be a complete noncompact Kähler manifold of complex dimension $n$ with nonnegative holomorphic bisectional curvature. Denote by $\mathcal{O}_{d}\left(M^{n}\right)$ the space of holomorphic functions of polynomial growth of degree at most $d$ on $M^{n}$. In this paper we prove that

$$
\operatorname{dim}_{\mathbb{C}} \mathcal{O}_{d}\left(M^{n}\right) \leq \operatorname{dim}_{\mathbb{C}} \mathcal{O}_{[d]}\left(\mathbb{C}^{n}\right),
$$

for all $d>0$, with equality for some positive integer $d$ if and only if $M^{n}$ is holomorphically isometric to $\mathbb{C}^{n}$. We also obtain sharp improved dimension estimates when its volume growth is not maximal or its Ricci curvature is positive somewhere.
\end{abstract}

\section{INTRODUCTION}

In this paper we discuss the function theory of complete manifolds with nonnegative curvature. Recall that the classical Liouville theorem states that any bounded (or even just positive) harmonic function is constant on Euclidean space. In [22], Yau extended the classical Liouville theorem to complete noncompact Riemannian manifolds with nonnegative Ricci curvature. It was further shown by Cheng and Yau in [4 that any harmonic function with sublinear growth on a complete noncompact Riemannian manifold with nonnegative Ricci curvature must be constant. For the space consisting of all harmonic functions of polynomial growth of degree at most $d$ on a complete noncompact Riemanian manifold $M^{m}$ of real dimension $m$ with nonnegative Ricci curvature, denoted by $\mathcal{H}_{d}\left(M^{m}\right)$, Yau asked in 23 if the dimension of $\mathcal{H}_{d}\left(M^{m}\right)$ is finite for each positive integer $d$, and if there holds

$$
\operatorname{dim}_{\mathbb{R}} \mathcal{H}_{d}\left(M^{m}\right) \leq \operatorname{dim}_{\mathbb{R}} \mathcal{H}_{d}\left(\mathbb{R}^{m}\right)
$$

for each positive integer $d$, with equality for some positive integer $d$ if and only if $M^{m}$ is isometric to $\mathbb{R}^{m}$. The first question was affirmativelly answered by Colding and Minicozzi [3], and in [12] Li produced an elegant short proof. For the latter question, the sharp upper bound estimate is still missing except for the special cases $m=2$ or $d=1$ obtained by Li-Tam [14, [15] and Kasue [10], and the rigidity part is only known for the special case $d=1$ obtained by Li [11] and CheegerColding-Minicozzi [2]. On the other hand, the example of Donnelly [8] shows that

Received by the editors October 1, 2003.

2000 Mathematics Subject Classification. Primary 32Q30; Secondary 32Q10, 32Q15.

The first author was partially supported by NSFC 10401042 and FANEDD 200216. The second author was partially supported by NSFC 10171114. The last author was partially supported by NSFC 10428102. 
one cannot expect the above sharp dimension estimates to have the stronger version

$$
\operatorname{dim}_{\mathbb{R}} \mathcal{H}_{d}\left(M^{m}\right) \leq \operatorname{dim}_{\mathbb{R}} \mathcal{H}_{[d]}\left(\mathbb{R}^{m}\right)
$$

for each positive real number $d$, where $[d]$ is the greatest integer less than or equal to $d$.

In complex geometry, geometers studied (holomorphic) function theory with the primary reason to prove a uniformization-type theorem in higher dimensions. In 20], Siu-Yau initiated a program of using holomorphic functions of polynomial growth to holomorphically embed a complete Kähler manifold into complex Euclidean spaces in an effort to generalize the classical uniformization theorem. The well-known uniformization conjecture for parabolic manifolds asks if every complete noncompact Kähler manifold with nonnegative holomorphic bisectional curvature is biholomorphic to a complex vector bundle over a compact Hermitian symmetric space. For this sake, Yau [23] proposed to study the spaces of holomorphic functions of polynomial growth on complete noncompact Kähler manifolds with nonnegative holomorphic bisectional curvature. Let us denote by $\mathcal{O}_{d}\left(M^{n}\right)$ the space of holomorphic functions of polynomial growth of degree at most $d$ on a complete noncompact Kähler manifold $M^{n}$ of complex dimension $n$ with nonnegative holomorphic bisectional curvature. The complex counterpart of the above Yau's conjecture states that there holds

$$
\operatorname{dim}_{\mathbb{C}} \mathcal{O}_{d}\left(M^{n}\right) \leq \operatorname{dim}_{\mathbb{C}} \mathcal{O}_{d}\left(\mathbb{C}^{n}\right)
$$

for each positive integer $d$, with equality for some positive integer $d$ if and only if $M^{n}$ is holomorphically isometric to $\mathbb{C}^{n}$. In a recent beautiful work [18, Ni obtained the following stronger version of dimension comparison result under an additional condition that the Kähler manifold $M^{n}$ is of maximum volume growth.

Theorem 1.1 (Ni [18). Let $M^{n}$ be a complete noncompact Kähler manifold of complex dimension $n$ with nonnegative holomorphic bisectional curvature. Assume that $M^{n}$ is of maximum volume growth, i.e.,

$$
\operatorname{Vol}\left(B\left(x_{0}, r\right)\right) \geq c r^{2 n}, \quad \text { for all } 0 \leq r<+\infty
$$

for some point $x_{0} \in M^{n}$ and some positive constant $c$. Then

$$
\operatorname{dim}_{\mathbb{C}} \mathcal{O}_{d}\left(M^{n}\right) \leq \operatorname{dim}_{\mathbb{C}} \mathcal{O}_{[d]}\left(\mathbb{C}^{n}\right), \quad \text { for each } d>0,
$$

where $[d]$ is the greatest integer less than or equal to $d$.

Our first result in this paper is the following theorem which resolves the remaining cases for the dimension comparison and establishes the rigidity.

Theorem 1.2. Let $M^{n}$ be a complete noncompact Kähler manifold of complex dimension $n$ with nonnegative holomorphic bisectional curvature. Then there holds

$$
\operatorname{dim}_{\mathbb{C}} \mathcal{O}_{d}\left(M^{n}\right) \leq \operatorname{dim}_{\mathbb{C}} \mathcal{O}_{[d]}\left(\mathbb{C}^{n}\right), \quad \text { for each } d>0 .
$$

Moreover the equality holds for some positive integer $d$ if and only if $M^{n}$ is holomorphically isometric to the complex Euclidean space $\mathbb{C}^{n}$ with the standard flat metric.

Our proof of this theorem is inspired by the recent work [18]. In [18, Ni obtained a Li-Yau-Hamilton type inequality and interpreted it as Bishop-Lelong monotonicity on complete Kähler manifolds with nonnegative bisectional curvature. Under the maximal volume growth assumption, $\mathrm{Ni}$ [18] showed that this monotonicity implies a bound of the vanishing order of a holomorphic function at a fixed point 
by its degree of polynomial growth. We observe that this estimate actually holds for the general case. Thus by a standard Poincaré-Siegel argument, this will give the sharp dimension estimates. For the rigidity part, we will use the equality of the sharp dimension estimate for some positive integer to force the Li-Yau-Hamilton type inequality to become an equality and then apply the strong maximum principle and the Laplacian comparison theorem to conclude that the manifold must be Euclidean. These arguments are reminiscent of some recent developments of the Ricci flows by Hamilton [9, Cao [1, Chen-Zhu [5] and Ni [17, where the equality in the Li-Yau-Hamilton inequality of the Ricci flows was used to characterize the type II and type III singularity models as steady and expanding Ricci solitons.

We next try to improve the above dimension estimates when the Kähler manifolds are not isometric to the flat complex Euclidean space $\mathbb{C}^{n}$. The second result of this paper is the following.

Theorem 1.3. Let $M^{n}$ be a complete noncompact Kähler manifold of complex dimension $n$ with nonnegative holomorphic bisectional curvature. Assume that the Ricci curvature of $M^{n}$ is positive at least at one point in $M^{n}$. Then there exists a positive constant $\epsilon \in(0,1)$ such that

$$
\operatorname{dim}_{\mathbb{C}} \mathcal{O}_{d}\left(M^{n}\right) \leq \operatorname{dim}_{\mathbb{C}} \mathcal{O}_{[(1-\epsilon) d]}\left(\mathbb{C}^{n}\right)
$$

for any positive integer $d>0$.

We remark that the positive constant $\epsilon$ is depending on the manifold. In Section 3 , we will present a family of complete noncompact Kähler manifolds with positive holomorphic bisectional curvature such that for each $\epsilon \in(0,1)$, there exists an element of this family such that the equality in the above Theorem 1.3 holds for each positive integer $d$. We also remark that the assumption that the Ricci curvature is positive at least at one point in $M^{n}$ is almost necessary. In fact, by considering the product manifold $\mathbb{C}^{p} \times M_{2}^{n-p}$ with $1 \leq p \leq n$, we have $\operatorname{dim}_{\mathbb{C}} \mathcal{O}_{1}\left(\mathbb{C}^{p} \times M_{2}^{n-p}\right) \geq p+1$, but $\operatorname{dim}_{\mathbb{C}} \mathcal{O}_{[1-\epsilon]}\left(\mathbb{C}^{n}\right)=1$ for any $\epsilon>0$.

The third result of this paper is to estimate the dimension in terms of the volume growth and to establish the corresponding rigidity.

Theorem 1.4. Let $M^{n}$ be a complete noncompact Kähler manifold of complex dimension $n$ with nonnegative holomorphic bisectional curvature. Suppose there exist $x_{0} \in M$, and $k>0, C>0$ such that,

$$
\operatorname{Vol}\left(B\left(x_{0}, r\right)\right) \leq C(1+r)^{2 k}, \quad \text { for all } r \geq 0 .
$$

Then there holds

$$
\operatorname{dim}_{\mathbb{C}} \mathcal{O}_{d}\left(M^{n}\right) \leq \operatorname{dim}_{\mathbb{C}} \mathcal{O}_{[d]}\left(\mathbb{C}^{[k]}\right), \quad \text { for each } d>0 .
$$

Moreover the equality holds for some positive integer $d$ if and only if $M^{n}$ is holomorphically isometric to $\mathbb{C}^{[k]} \times M_{2}^{n-[k]}$ for some complete Kähler manifold $M_{2}^{n-[k]}$ of complex dimension $n-[k]$ with nonnegative holomorphic bisectional curvature which carries no nontrivial holomorphic functions of polynomial growth.

Observe that the assumption on the curvature and the Bishop volume comparison theorem asserts that

$$
\operatorname{Vol}\left(B\left(x_{0}, r\right)\right) \leq \omega_{2 n} r^{2 n}, \quad \text { for all } r \geq 0,
$$

where $\omega_{2 n}$ is the volume of the unit ball in $\mathbb{R}^{2 n}$. On the other hand, a theorem of Calabi and Yau 21] asserts that $\operatorname{Vol}\left(B\left(x_{0}, r\right)\right)$ must grow at least linearly. Hence 
the constant $k$ in Theorem 1.4 must exist and satisfy $1 \leq k \leq n$. In particular, Theorem 1.2 is a special case of Theorem 1.4. The real counterpart of Theorem 1.4 for harmonic functions of polynomial growth had been studied by Li-Tam [14, [15], Kasue [10], Li [11] and Cheeger-Colding-Minicozzi [2] for the special cases $d=1$ or real dimension $m=2$. However the general case remains as open questions (see for example Question 9.6 and Question 9.7 in [13]).

We organize the paper as follows: in Section 2 we prove Theorem 1.2; in Section 3 we prove a slightly generalized version of Theorem 1.3; finally in Section 4 we give a proof of Theorem 1.4.

\section{SharP DIMENSION ESTIMATE AND RIGIDITY}

Let $M^{n}$ be a complete noncompact Kähler manifold of complex dimension $n$. Fix a point $x_{0} \in M^{n}$. We call a holomorphic function $f$ of polynomial growth if there exists $d \geq 0$ and $C$ depending on $x_{0}, d$ and $f$ such that

$$
|f(x)| \leq C\left(1+r^{d}\left(x, x_{0}\right)\right), \quad \forall x \in M^{n},
$$

where $r\left(x, x_{0}\right)$ is the distance between $x$ and $x_{0}$. For a holomorphic function $f$ of polynomial growth on $M^{n}$, we define the degree of $f$ by

$$
\operatorname{deg}(f)=\inf \left\{d \mid \begin{array}{c}
|f(x)| \leq C\left(1+r^{d}\left(x, x_{0}\right)\right), \quad \forall x \in M^{n}, \\
\text { for some } d \geq 0 \text { and } C=C\left(d, x_{0}, f\right)
\end{array}\right\} .
$$

It is clear that the definition of $\operatorname{deg}(f)$ is independent of the choice of the point $x_{0}$ in $M^{n}$. For any $d \geq 0$ we denote by $\mathcal{O}_{d}\left(M^{n}\right)$ the complex linear space of all holomorphic functions of polynomial growth of degree at most $d$ on the Kähler manifold $M^{n}$.

For any $f \in \mathcal{O}_{d}\left(M^{n}\right)$, we consider the following heat equation:

$$
\left\{\begin{array}{l}
\left(\frac{\partial}{\partial t}-\Delta\right) u(x, t)=0, \quad x \in M^{n}, t>0, \\
u(x, 0)=\log |f(x)|^{2}, \quad x \in M^{n} .
\end{array}\right.
$$

Assuming the Kähler manifold $M^{n}$ has nonnegative Ricci curvature, it is shown by $\mathrm{Ni}$ (see Lemma 3.1 in [18]) that the equation (2.1) admits a smooth solution $u(x, t)$ on $M^{n} \times(0,+\infty)$ and the solution $u(x, t)$ is given by

$$
u(x, t)=\int_{M} H(x, y, t) \log |f(y)|^{2} d y, \quad \forall x \in M, t>0,
$$

where $H(x, y, t)$ is the heat kernel of $M^{n}$.

Lemma 2.1. Let $M^{n}$ be a complete noncompact Kähler manifold with nonnegative Ricci curvature and let $u(x, t)$ solve the heat equation (2.1). Then

$$
\limsup _{t \rightarrow+\infty} \frac{u(x, t)}{\log t} \leq d
$$

Proof. We write the solution $u(x, t)$ in terms of the heat kernel as

$$
\begin{aligned}
u(x, t) & =\int_{M} H(x, y, t) \log |f(y)|^{2} d y \\
& =\int_{\{r(x, y) \leq \sqrt{t}\}} H(x, y, t) \log |f(y)|^{2} d y+\int_{\{r(x, y)>\sqrt{t}\}} H(x, y, t) \log |f(y)|^{2} d y,
\end{aligned}
$$


where $r(x, y)$ is the distance function from the point $x \in M^{n}$. Since $f \in \mathcal{O}_{d}\left(M^{n}\right)$, for arbitrary $\epsilon>0$, there exists a positive constantsSharp dimension estimates $C=C(d, \epsilon, x, f)$ such that

$$
|f(y)| \leq C\left(1+r^{d+\epsilon}(x, y)\right), \quad \forall y \in M^{n} .
$$

Then we have for $t>1$,

$$
\begin{aligned}
u(x, t) \leq & \int_{\{r(x, y) \leq \sqrt{t}\}} H(x, y, t)\left((d+\epsilon) \log t+C_{1}\right) d y \\
& +\int_{\{r(x, y)>\sqrt{t}\}} H(x, y, t)\left((d+\epsilon) \log ^{2}(x, y)+C_{2}\right) d y \\
\leq & (d+\epsilon) \log t+C_{3}+(d+\epsilon) \int_{\{r(x, y)>\sqrt{t}\}} H(x, y, t) \log \left(\frac{r^{2}(x, y)}{t}\right) d y
\end{aligned}
$$

for some positive constant $C_{1}, C_{2}, C_{3}$ depending only on $d, \epsilon, x$ and $f$. Here we have used the fact that $\int_{M^{n}} H(x, y, t) d y=1$.

By applying the heat kernel estimate of Li-Yau [16] and the standard volume comparison, there exist positive constant $C(n)$ and $\tilde{C}(n)$ depending only on the dimension such that

$$
\begin{aligned}
& \int_{\{r(x, y)>\sqrt{t}\}} H(x, y, t) \log \left(\frac{r^{2}(x, y)}{t}\right) d y \\
\leq & C(n) \int_{\{r(x, y)>\sqrt{t}\}} \frac{1}{\operatorname{Vol}(B(x, \sqrt{t}))} \exp \left(-\frac{r^{2}(x, y)}{5 t}\right) \cdot \log \left(\frac{r^{2}(x, y)}{t}\right) d y \\
\leq & C(n) \sum_{k=0}^{\infty} \int_{\left\{2^{k+1} \sqrt{t} \geq r(x, y) \geq 2^{k} \sqrt{t}\right\}} \frac{1}{\operatorname{Vol}(B(x, \sqrt{t}))} \exp \left(-\frac{r^{2}(x, y)}{5 t}\right) \cdot \log \left(\frac{r^{2}(x, y)}{t}\right) d y \\
\leq & C(n) \sum_{k=0}^{\infty} \exp \left(-\frac{2^{2 k}}{5}\right) \cdot \log 2^{2(k+1)} \cdot\left(2^{k+1}\right)^{2 n} \\
\leq & \tilde{C}(n) .
\end{aligned}
$$

Hence we have for $t>1$,

$$
u(x, t) \leq(d+\epsilon) \log t+C_{3}+(d+\epsilon) \tilde{C}(n),
$$

which completes the proof of Lemma 2.1.

For a nontrivial holomorphic function $f \in \mathcal{O}_{d}\left(M^{n}\right)$, the vanishing order of $f$ at a fixed point $x \in M^{n}$ is defined by

$$
\operatorname{ord}_{x}(f)=\max \left\{m\left|D^{\alpha} f(x)=0, \forall\right| \alpha \mid<m\right\} .
$$

The following result was obtained by $\mathrm{Ni}$ in 18 .

Lemma 2.2 ( $\mathrm{Ni}[18)$. Let $M^{n}$ be a complete noncompact Kähler manifold with nonnegative holomorphic bisectional curvature. Also, let $u(x, t), x \in M$ and $t \in$ 
$(0,+\infty)$, be the solution of heat equation (2.1) with $f \in \mathcal{O}_{d}\left(M^{n}\right)$ a nontrivial holomorphic function. Denote $w(x, t)=\frac{\partial}{\partial t} u(x, t)$. Then

$$
\lim _{t \rightarrow 0} t w(x, t)=\operatorname{ord}_{x}(f) .
$$

We are now ready to prove Theorem 1.2.

Proof of Theorem 1.2. Let $M^{n}$ be a complete noncompact Kähler manifold of complex dimension $n$ with nonnegative holomorphic bisectional curvature. For any nontrivial $f \in \mathcal{O}_{d}\left(M^{n}\right)$, let $u(x, t)$, on $M^{n} \times(0,+\infty)$, be the solution of the heat equation (2.1). When we consider the trivial line bundle $M^{n} \times \mathbb{C} \rightarrow M^{n}$ equipped with the metric $e^{-u(x, t)}$ on the fibers, the complex Hessian $u_{i \bar{j}}$ corresponds to the bundle curvature tensor, and the heat equation (2.1) is exactly the Hermitian YangMills flow for Hermitian metric.

Differentiating the equation (2.1) we have for normal coordinates at a point

$$
\left(u_{t}\right)_{i \bar{j}}=R_{l \bar{k} i \bar{j}} u_{k \bar{l}}+\frac{\partial^{4} u}{\partial z^{k} \partial \bar{z}^{k} \partial z^{i} \partial \bar{z}^{j}}
$$

and by definition and an easy computation

$$
\begin{aligned}
\Delta u_{i \bar{j}} & =\frac{1}{2}\left(u_{i \bar{j}, k \bar{k}}+u_{i \bar{j}, \bar{k} k}\right) \\
& =\frac{\partial^{4} u}{\partial z^{i} \partial \bar{z}^{j} \partial z^{k} \partial \bar{z}^{k}}+\frac{1}{2}\left(R_{i \bar{l}} u_{l \bar{j}}+R_{l \bar{j}} u_{i \bar{l}}\right) .
\end{aligned}
$$

Thus the bundle curvature tensor $u_{i \bar{j}}$ satisfies the complex Lichnerowicz-Laplacian heat equation

$$
\left(\frac{\partial}{\partial t}-\Delta\right) u_{i \bar{j}}=R_{l \bar{k} i \bar{j}} u_{k \bar{l}}-\frac{1}{2}\left(R_{i \bar{l}} u_{l \bar{j}}+R_{l \bar{j}} u_{i \bar{l}}\right) .
$$

It was shown by $\mathrm{Ni}[18$ ] (Lemma 2.1 in [18]) that the nonnegativity of the bundle curvature tensor $u_{i \bar{j}}$ is preserved for all $t>0$. More importantly, Ni [18] (Corollary 2.2 in [18]) proved that the Hermitian Yang-Mills flow possesses a Li-Yau-Hamilton inequality (as Ricci flow or mean curvature flow)

$$
Z(V) \triangleq w_{t}+\nabla_{k} w \cdot V^{k}+\nabla_{\bar{k}} w \cdot V^{\bar{k}}+u_{i \bar{j}} V^{i} V^{\bar{j}}+\frac{w}{t} \geq 0
$$

on $M^{n} \times(0,+\infty)$ for any $(1,0)$ vector field $V$. Here $w(x, t)=\frac{\partial}{\partial t} u(x, t)=\Delta u(x, t)$ is the trace of the bundle curvature tensor. In particular by choosing $V \equiv 0$,

$$
\frac{\partial}{\partial t}(t w(x, t)) \geq 0,
$$

on $M^{n} \times(0,+\infty)$.

We now follow an argument of $\mathrm{Ni}$ in 18 by using (2.5), Lemma 2.1 and Lemma 2.2 to derive a sharp bound on the vanishing order of the holomorphic function. For a large enough positive integer $p$, we have

$$
\begin{aligned}
u(x, t)=\int_{1}^{t} w(x, s) d s+u(x, 1) & \geq \int_{t^{\frac{1}{p}}}^{t} \frac{t^{\frac{1}{p}}}{s} w\left(x, t^{\frac{1}{p}}\right) d s+u(x, 1) \\
& =\left(1-\frac{1}{p}\right) \log t \cdot t^{\frac{1}{p}} w\left(x, t^{\frac{1}{p}}\right)+u(x, 1) .
\end{aligned}
$$


By combining with Lemma 2.1, we have

$$
\limsup _{t \rightarrow \infty}\left(1-\frac{1}{p}\right) t^{\frac{1}{p}} w\left(x, t^{\frac{1}{p}}\right) \leq d
$$

and by arbitrariness of $p$,

$$
\limsup _{t \rightarrow \infty} t w(x, t) \leq d
$$

So by combining with (2.5), we deduce

$$
t w(x, t) \leq d, \quad \text { for all } t \geq 0 .
$$

On the other hand, from Lemma 2.2,

$$
\lim _{t \rightarrow 0} t w(x, t)=\operatorname{ord}_{x}(f) .
$$

Hence we have the following sharp bound on vanishing order:

$$
\operatorname{ord}_{x}(f) \leq[d], \quad \text { for any nontrivial } f \in \mathcal{O}_{d}\left(M^{n}\right),
$$

since the vanishing order needs to be an integer.

We then perform the standard Poincaré-Siegel argument. For the fixed $x \in M^{n}$ and a local holomorphic coordinate system $\left\{z^{1}, \cdots, z^{n}\right\}$ near $x$, define the PoincaréSiegel map

$$
\begin{aligned}
P: \mathcal{O}_{d}\left(M^{n}\right) & \rightarrow \mathbb{C}^{k_{[d]}} \\
f & \longmapsto\left(f(x), D f(x), \cdots, D^{\alpha} f(x), \cdots\right), \quad \text { for all }|\alpha| \leq[d],
\end{aligned}
$$

where $k_{[d]}=\operatorname{dim}_{\mathbb{C}}\left(\mathcal{O}_{[d]}\left(\mathbb{C}^{n}\right)\right)$. The inequality (2.7) implies that the map $P$ must be injective. Thus we have proved

$$
\operatorname{dim}_{\mathbb{C}} \mathcal{O}_{d}\left(M^{n}\right) \leq \operatorname{dim}_{\mathbb{C}} \mathcal{O}_{[d]}\left(\mathbb{C}^{n}\right)
$$

for each positive real number $d$.

From now on, we study the rigidity part of Theorem 1.2. Suppose there exists a positive integer $d$ such that

$$
\operatorname{dim}_{\mathbb{C}} \mathcal{O}_{d}\left(M^{n}\right)=\operatorname{dim}_{\mathbb{C}} \mathcal{O}_{d}\left(\mathbb{C}^{n}\right) .
$$

From the above argument we know that there exists a nonconstant function $f \in$ $\mathcal{O}_{d}\left(M^{n}\right)$ and a point $x_{0} \in M^{n}$ such that

$$
\operatorname{ord}_{x_{0}}(f)=d \text {. }
$$

Let $u(x, t)$ be the solution of the heat equation (2.1) with the initial data $\log |f|^{2}$, and let $w(x, t)=\frac{\partial}{\partial t} u(x, t)=\Delta u(x, t)$. By (2.6) and Lemma 2.2, we have

$$
t w\left(x_{0}, t\right) \equiv d, \quad \text { for all } t>0,
$$

which implies

$$
Z(0)=\left(w_{t}+\frac{w}{t}\right)\left(x_{0}, t\right) \equiv 0, \quad \text { for all } t>0 .
$$

In the following we distinguish two cases. Case (i): $\left(u_{i \bar{j}}(x, t)\right)$ is positive definite on $M^{n} \times(0,+\infty)$ and Case (ii): $\left(u_{i \bar{j}}(x, t)\right)$ has nontrivial kernel for some $(x, t) \in$ $M^{n} \times(0,+\infty)$.

Let us first consider Case (i). Recall from (2.4) that

$$
Z(V) \triangleq w_{t}+\frac{w}{t}+\nabla_{k} w \cdot V^{k}+\nabla_{\bar{k}} w \cdot V^{\bar{k}}+u_{i \bar{j}} V^{i} V^{\bar{j}} \geq 0
$$


on $M^{n} \times(0,+\infty)$ for any $(1,0)$ vector field $V$. Since $\left(u_{i \bar{j}}\right)>0$, we see that there exists a unique smooth vector field $V$ which minimizes $Z$ and satisfies

$$
\nabla_{k} w+u_{k \bar{j}} V^{\bar{j}}=0, \quad \text { everywhere, }
$$

by the first variation formula. We then consider the function $Z=Z(V)$ by taking $V$ to be the unique minimizing vector field. It was shown by $\mathrm{Ni}$ in Lemma 1.3 of [18] that the function $Z$ satisfies, for normal coordinates at a point,

$$
\begin{aligned}
\left(\frac{\partial}{\partial t}-\Delta\right) Z= & R_{i \bar{j} k \bar{l}} u_{l \bar{k}} V^{i} V^{\bar{j}}+u_{i \bar{j}}\left(\nabla_{\bar{k}} V_{j}-\frac{1}{t} g_{j \bar{k}}\right)\left(\nabla_{k} V_{\bar{i}}-\frac{1}{t} g_{k \bar{i}}\right) \\
& +u_{i \bar{j}} \nabla_{\bar{k}} V_{\bar{i}} \nabla_{k} V_{j}-\frac{2 Z}{t} .
\end{aligned}
$$

Note that the bisectional curvature is nonnegative, $u$ is plurisubharmonic and by (2.9) $Z\left(x_{0}, t\right)=0$ for all $t>0$. Thus by the strong maximum principle, we see that the function $Z$ must be identically zero everywhere. And from (2.10) we have

$$
\begin{gathered}
R_{i \bar{j} k \bar{l}} u_{l \bar{k}} V^{i} V^{\bar{j}}=0, \\
u_{i \bar{j}}\left(\nabla_{\bar{k}} V_{j}-\frac{1}{t} g_{j \bar{k}}\right)\left(\nabla_{k} V_{\bar{i}}-\frac{1}{t} g_{k \bar{i}}\right)=0, \\
u_{i \bar{j}} \nabla_{\bar{k}} V_{\bar{i}} \nabla_{k} V_{j}=0,
\end{gathered}
$$

everywhere on $M^{n} \times(0,+\infty)$. Since we assumed that $\left(u_{i \bar{j}}(x, t)\right)$ is positive definite everywhere in Case (i), we have from (2.12) and (2.13),

$$
\left\{\begin{array}{l}
\nabla_{i} V_{\bar{j}}=\nabla_{\bar{j}} V_{i}=\frac{1}{t} g_{i \bar{j}}, \\
\nabla_{i} V_{j}=\nabla_{\bar{i}} V_{\bar{j}}=0 .
\end{array}\right.
$$

Let us first assume that the manifold $M^{n}$ is simply connected. Then there exists a function $\varphi$ on $M^{n}$ such that

$$
\left\{\begin{array}{l}
\varphi_{i \bar{j}}=g_{i \bar{j}}, \\
\nabla_{i} \nabla_{j} \varphi=\nabla_{\bar{i}} \nabla_{\bar{j}} \varphi=0 .
\end{array}\right.
$$

Note that for any normal geodesic $\gamma$, we have

$$
\frac{d^{2}}{d s^{2}} \varphi \circ \gamma(s)=\operatorname{Hess}(\varphi)\left(\gamma^{\prime}, \gamma^{\prime}\right)=1
$$

This implies that $\varphi$ is a proper convex function. Let $p_{0} \in M^{n}$ be the unique minimizer of $\varphi$. We may assume $\varphi\left(p_{0}\right)=0$. Then from (2.15) it is clear that

$$
\varphi(x)=\frac{1}{2} r^{2}\left(x, p_{0}\right), \quad \text { on } M^{n} .
$$

Hence by (2.14) and the rigidity part of the standard Hessian comparison theorem (or Laplacian comparison theorem), we know that $M^{n}$ is flat and then $M$ is holomorphically isometric to $\mathbb{C}^{n}$ since $M^{n}$ is assumed to be simply connected.

To remove the simply connectedness assumption, we note that

$$
\operatorname{dim}_{\mathbb{C}} \mathcal{O}_{d}\left(M^{n}\right) \leq \operatorname{dim}_{\mathbb{C}} \mathcal{O}_{d}\left(\tilde{M}^{n}\right),
$$

where $\tilde{M}^{n}$ is the universal cover of $M^{n}$. Thus from (2.18) and the assumption on $d$, we have

$$
\operatorname{dim}_{\mathbb{C}} \mathcal{O}_{d}\left(\tilde{M}^{n}\right)=\operatorname{dim}_{\mathbb{C}} \mathcal{O}_{d}\left(\mathbb{C}^{n}\right)
$$


and then by the above argument, $\tilde{M}^{n}$ is holomorphically isometric to $\mathbb{C}^{n}$. Note that every function in $\mathcal{O}_{d}\left(M^{n}\right)$ can be lifted as a function of $\mathcal{O}_{d}\left(\tilde{M}^{n}\right)$. This means that the complex linear space $\mathcal{O}_{d}\left(M^{n}\right)$ is a subspace of $\mathcal{O}_{d}\left(\tilde{M}^{n}\right)$. Since

$$
\operatorname{dim}_{\mathbb{C}} \mathcal{O}_{d}\left(M^{n}\right)=\operatorname{dim}_{\mathbb{C}} \mathcal{O}_{d}\left(\tilde{M}^{n}\right)=\operatorname{dim}_{\mathbb{C}} \mathcal{O}_{d}\left(\mathbb{C}^{n}\right)
$$

we see that every function in $\mathcal{O}_{d}\left(\tilde{M}^{n}\right)$ is $\pi_{1}\left(M^{n}\right)$-invariant. Let $z^{1}, \cdots, z^{n}$ be the coordinate functions on $\tilde{M}^{n}=\mathbb{C}^{n}$. Obviously $z^{1}, \cdots, z^{n} \in \mathcal{O}_{d}\left(\tilde{M}^{n}\right)$. Let $\sigma \in \pi_{1}\left(M^{n}\right)$ be a deck transformation. Since the functions $z^{1}, \cdots, z^{n}$ are $\pi_{1}\left(M^{n}\right)$ invariant, we have

$$
\sigma\left(\left[z^{i}=0\right]\right)=\left[z^{i}=0\right], \quad \text { for } i=1, \cdots, n .
$$

Hence

$$
\sigma(\{0\})=\sigma\left(\bigcap_{i=1}^{n}\left[z^{i}=0\right]\right)=\bigcap_{i=1}^{n}\left[z^{i}=0\right]=\{0\} .
$$

This implies $\pi_{1}\left(M^{n}\right)=0$, so we have finished the proof of Case (i).

We next consider Case (ii). The argument of the previous paragraph tells us that we may assume $M^{n}$ is simply connected. Recall that $\left(u_{i \bar{j}}(x, t)\right)$ is nonnegative definite on $M^{n} \times(0,+\infty)$ and satisfies the complex Lichnerowicz-Laplacian heat equation (2.3). Suppose that the matrix $\left(u_{i \bar{j}}(x, t)\right)$ has nontrivial kernel at some $(\bar{x}, \bar{t}) \in M^{n} \times(0,+\infty)$. Then by $(2.3)$ and the strong maximum principle, we know that for all $t<\bar{t}$ and $x \in M^{n},\left(u_{i \bar{j}}(x, t)\right)$ has nontrivial kernel. Denote the kernel space of $\left(u_{i \bar{j}}(x, t)\right)$ by $K(x, t) \subset T_{x}^{1,0} M^{n}$. It was shown by Ni-Tam in Corollary 2.1 of [19] that there exists $\tilde{t} \in(0, \bar{t})$ such that for any $0<t<\tilde{t}, K(x, t)$ is a distribution which is invariant under parallel translations. Moreover the Kähler manifold $M^{n}$ splits isometrically and holomorphically as

$$
M^{n}=M_{1}^{p} \times M_{2}^{n-p}
$$

with $1 \leq p \leq n$, where $K$ corresponds the tangent bundle of $M_{1}^{p}$ and $\left(u_{i \bar{j}}(x, t)\right)>$ 0 on $M_{2}^{n-p} \times(0, \tilde{t})$. Both $M_{1}^{p}$ and $M_{2}^{n-p}$ are complete Kähler manifolds with nonnegative holomorphic bisectional curvature.

Write $x=\left(x_{1}, x_{2}\right) \in M^{n}=M_{1}^{p} \times M_{2}^{n-p}$ with $x_{1} \in M_{1}^{p}$ and $x_{2} \in M_{2}^{n-p}$. Since the restriction $\left.u_{i \bar{j}}\right|_{M_{1}^{p} \times(0, \tilde{t})} \equiv 0$, we have that for any fixed $x_{2} \in M_{2}^{n-p}$ and $t \in(0, \tilde{t})$, the function $u\left(\cdot, x_{2}, t\right)$ is a pluriharmonic function on $M_{1}^{p}$. This is,

$$
d_{x_{1}} d_{x_{1}}^{c} u\left(\cdot, x_{2}, t\right)=0, \quad \text { on } M_{1}^{p},
$$

where $d_{x_{1}}$ is the exterior differential on $M_{1}^{p}$ and $d_{x_{1}}^{c}=\sqrt{-1}\left(\bar{\partial}_{x_{1}}-\partial_{x_{1}}\right)$ is the usual real operator. By the heat kernel estimate of Li-Yau [16, it is not hard (see for example Corollary 1.4 in [19]) to see that the function $u\left(\cdot, x_{2}, t\right)$ is at most upper logarithmic growth on $M_{1}^{p}$ (but we cannot ensure such a lower bound). Since we assumed $M^{n}$ is simply connected, $M_{1}^{p}$ is also simply connected. Thus there exists a real function $v$ on $M_{1}^{p}$ such that

$$
d_{x_{1}}^{c} u\left(\cdot, x_{2}, t\right)=d_{x_{1}} v(\cdot), \quad \text { on } M_{1}^{p}
$$

i.e.,

$$
\sqrt{-1}\left(\bar{\partial}_{x_{1}}-\partial_{x_{1}}\right) u\left(\cdot, x_{2}, t\right)=\left(\partial_{x_{1}}+\bar{\partial}_{x_{1}}\right) v(\cdot), \quad \text { on } M_{1}^{p}
$$

which implies

$$
\bar{\partial}_{x_{1}}\left(u\left(\cdot, x_{2}, t\right)+\sqrt{-1} v(\cdot)\right)=0, \quad \text { on } M_{1}^{p} \text {. }
$$


So $u\left(\cdot, x_{2}, t\right)+\sqrt{-1} v(\cdot)$ is a holomorphic function on $M_{1}^{p}$. Let $\alpha>0$ be a positive constant and set

$$
F(\cdot)=e^{\alpha\left(u\left(\cdot, x_{2}, t\right)+\sqrt{-1} v(\cdot)\right)}, \quad \text { on } M_{1}^{p} .
$$

Clearly the function $F$ is holomorphic on $M_{1}^{p}$. When we choose $\alpha>0$ small enough, there holds

$$
\left|F\left(x_{1}\right)\right| \leq C\left(1+r\left(x_{1}, x_{0}^{(1)}\right)\right)^{\frac{1}{2}}
$$

for all $x_{1} \in M_{1}^{p}$ and for some fixed $x_{0}^{(1)} \in M_{1}^{p}$, since the function $u\left(\cdot, x_{2}, t\right)$ is at most upper logarithmic growth on $M_{1}^{p}$. It then follows from Cheng-Yau [4] (or the vanishing order estimate (2.7)) that $F$ is constant on $M_{1}^{p}$. This implies that $u\left(\cdot, x_{2}, t\right)$ is constant on $M_{1}^{p}$. Hence the solution $u(x, t)$ can be regarded as a solution of the heat equation $(2.1)$ on $M_{2}^{n-p} \times(0, \tilde{t})$ with positive definite $\left(u_{i \bar{j}}(x, t)\right)$. Clearly the solution $u(x, t)$ is actually defined on $M_{2}^{n-p} \times(0,+\infty)$, and $\left(u_{i \bar{j}}(x, t)\right)$ is positive definite everywhere (by the strong maximum principle) on $M_{2}^{n-p} \times(0,+\infty)$.

The equation (2.9) tells us the function $w(x, t)=\frac{\partial}{\partial t} u(x, t)=\Delta u(x, t)$ satisfies

$$
Z(0)=\left(w_{t}+\frac{w}{t}\right)\left(x_{0}, t\right) \equiv 0, \quad \text { for all } t>0,
$$

for some $x_{0} \in M_{2}^{n-p}$. Then by repeating the argument of Case (i) we conclude that $M_{2}^{n-p}$ is holomorphically isometric to $\mathbb{C}^{n-p}$, so

$$
M^{n}=M_{1}^{p} \times \mathbb{C}^{n-p},
$$

isometrically and holomorphically. Since the function $f \in \mathcal{O}_{d}\left(M^{n}\right)$ with $\operatorname{ord}_{x_{0}}(f)=$ $d>0$ is nonconstant, it is clear from the strong maximum principle (see for example Lemma 3.1 in [18) that $w(x, t)>0$ everywhere on $M^{n} \times(0,+\infty)$. This says the rank of $\left(u_{i \bar{j}}(x, t)\right)$ is at least 1 . Hence we must have $p \leq n-1$.

We now claim that

$$
\operatorname{dim}_{\mathbb{C}} \mathcal{O}_{d}\left(M_{1}^{p}\right)=\operatorname{dim}_{\mathbb{C}} \mathcal{O}_{d}\left(\mathbb{C}^{p}\right) .
$$

Clearly once this is proved, then by induction on the dimension of the manifolds we will complete the proof of the rigidity part of Theorem 1.2.

Fix a point $\left(p_{0}, q_{0}\right) \in M_{1}^{p} \times \mathbb{C}^{n-p}$ and a local holomorphic coordinate system $\left(z_{1}, z_{2}\right)$ near the point $\left(p_{0}, q_{0}\right)$, where $z_{1}=\left(z_{1}^{1}, \cdots, z_{1}^{p}\right) \in M_{1}^{p}$ and $z_{2}=$ $\left(z_{2}^{1}, \cdots, z_{2}^{n-p}\right) \in \mathbb{C}^{n-p}$. For any holomorphic function $g \in \mathcal{O}_{d}\left(M^{n}\right), g$ has a Taylor expansion near $\left(p_{0}, q_{0}\right)$. Let us denote $P_{d}(g)\left(z_{1}, z_{2}\right)$ to be the polynomial obtained by truncating the Taylor expansion up to order $d$, i.e.,

$$
g\left(z_{1}, z_{2}\right)=P_{d}(g)\left(z_{1}, z_{2}\right)+\text { higher order terms. }
$$

Consider the Poincaré-Siegel map

$$
\begin{aligned}
P_{d}: \mathcal{O}_{d}\left(M^{n}\right) & \rightarrow \mathcal{O}_{d}\left(\mathbb{C}^{n}\right)=\mathcal{O}_{d}\left(\mathbb{C}^{p} \times \mathbb{C}^{n-p}\right), \\
g & \longmapsto P_{d}(g) .
\end{aligned}
$$

The estimate (2.7) implies that the map $P_{d}$ is injective. Since

$$
\operatorname{dim}_{\mathbb{C}} \mathcal{O}_{d}\left(M^{n}\right)=\operatorname{dim}_{\mathbb{C}} \mathcal{O}_{d}\left(\mathbb{C}^{n}\right)
$$

by the assumption, we see that the Poincaré-Siegel map $P_{d}$ is an isomorphism between $\mathcal{O}_{d}\left(M^{n}\right)$ and $\mathcal{O}_{d}\left(\mathbb{C}^{n}\right)$.

Since $M_{1}^{p}$ is also a complete Kähler manifold with nonnegative holomorphic bisectional curvature, we then also have the estimate (2.7) for any nontrivial $f \in$ 
$\mathcal{O}_{d}\left(M_{1}^{p}\right)$. Thus the corresponding Poincaré-Siegel map (by considering the Taylor expansion at $p_{0} \in M_{1}^{p}$ )

$$
P_{d}^{(1)}: \mathcal{O}_{d}\left(M_{1}^{p}\right) \rightarrow \mathcal{O}_{d}\left(\mathbb{C}^{p}\right)
$$

is also injective. To prove the claim of $(2.17)$, it suffices to prove that $P_{d}^{(1)}$ is surjective. Of course, we can regard $\mathcal{O}_{d}\left(M_{1}^{p}\right)$ as a complex subspace of $\mathcal{O}_{d}\left(M^{n}\right)$, and this Poincaré-Siegel map $P_{d}^{(1)}$ is just the restriction of the original $P_{d}$ on the subspace $\mathcal{O}_{d}\left(M_{1}^{p}\right)\left(\subset \mathcal{O}_{d}\left(M^{n}\right)\right)$. Since $P_{d}$ is a bijection between $\mathcal{O}_{d}\left(M^{n}\right)$ and $\mathcal{O}_{d}\left(\mathbb{C}^{n}\right)$, we only need to prove the assertion that if $g \in \mathcal{O}_{d}\left(M^{n}\right)$ with $P_{d}(g) \in \mathcal{O}_{d}\left(\mathbb{C}^{p}\right)\left(\subset \mathcal{O}_{d}\left(\mathbb{C}^{n}\right)\right)$, then $g \in \mathcal{O}_{d}\left(M_{1}^{p}\right)$. In fact, for such a $g$ with $P_{d}(g) \in \mathcal{O}_{d}\left(\mathbb{C}^{p}\right), g$ has a Taylor expansion of the form

$$
g\left(z_{1}, z_{2}\right)=P_{d}(g)\left(z_{1}\right)+\text { higher order terms }
$$

which implies that

$$
\left.D_{z_{2}}^{\alpha} g\left(z_{1}, z_{2}\right)\right|_{\left(p_{0}, q_{0}\right)}=0, \quad \text { for any }|\alpha| \leq d \text { and }|\alpha| \neq 0 .
$$

Fix $z_{1} \in M_{1}^{p}$ and consider the function $g\left(z_{1}, \cdot\right)$ on $\mathbb{C}^{n-p}$. Clearly the function $g\left(z_{1}, \cdot\right)$ belongs to $\mathcal{O}_{d}\left(\mathbb{C}^{n-p}\right)$ since $g$ belongs to $\mathcal{O}_{d}\left(M^{n}\right)$. Thus there also holds the vanishing order estimate (2.7). So by combining $(2.18)$ we see that $g\left(z_{1}, \cdot\right)$ is constant on $\mathbb{C}^{n-p}$. This says that $g$ is only a function of variable $z_{1} \in M_{1}^{p}$ and then $g \in \mathcal{O}_{d}\left(M_{1}^{p}\right)$.

Therefore we have proved the claim of (2.17) and have completed the proof of Theorem 1.2.

\section{IMPROVED DIMENSION ESTIMATE}

Let $M^{n}$ be a complete noncompact Kähler manifold of complex dimension $n$ with nonnegative holomorphic bisectional curvature. By inspecting the proof of the rigidity part of Theorem 1.2, we have actually proved that if there exists a holomorphic function $f$ of polynomial growth on $M^{n}$ such that

$$
\operatorname{ord}_{x}(f)=\operatorname{deg}(f)>0, \quad \text { for some } x \in M^{n},
$$

then the universal cover $\tilde{M}^{n}$ admits a splitting

$$
\tilde{M}^{n}=M_{1}^{p} \times \mathbb{C}^{n-p}
$$

isometrically and holomorphically for some $p \leq n-1$. Thus if $M^{n}$ does not admit its universal cover $\tilde{M}^{n}$ splitting isometrically and holomorphically as $\mathbb{C} \times M_{2}^{n-1}$, then we have

$$
\operatorname{ord}_{x}(f) \leq \operatorname{deg}(f)-1
$$

for all $f \in \mathcal{O}_{d}\left(M^{n}\right)$, all points $x \in M^{n}$ and all positive integers $d$. Consequently,

$$
\operatorname{dim}_{\mathbb{C}} \mathcal{O}_{d}\left(M^{n}\right) \leq \operatorname{dim}_{\mathbb{C}} \mathcal{O}_{d-1}\left(\mathbb{C}^{n}\right), \quad \text { for all positive integers } d .
$$

The following result improves this dimension estimate significantly as $d$ large.

Theorem 3.1. Let $M^{n}$ be a complete noncompact Kähler manifold of complex dimension $n$ with nonnegative holomorphic bisectional curvature. Suppose its universal cover $\tilde{M}^{n}$ does not admit a holomorphically isometric splitting as $\mathbb{C} \times M_{2}^{n-1}$. Then there exists a positive constant $\epsilon \in(0,1)$ such that

$$
\operatorname{dim}_{\mathbb{C}} \mathcal{O}_{d}\left(M^{n}\right) \leq \operatorname{dim}_{\mathbb{C}} \mathcal{O}_{[(1-\epsilon) d]}\left(\mathbb{C}^{n}\right), \quad \text { for all positive integers } d .
$$


Before giving the proof of Theorem 3.1, we remark that Theorem 1.3 in the Introduction is a consequence of the above Theorem 3.1. Indeed if a Kähler manifold $M^{n}$ admits its universal cover $\tilde{M}^{n}$ isometrically splitting as $\mathbb{C} \times M_{2}^{n-1}$, then its Ricci curvature must have a nontrivial kernel everywhere.

Proof of Theorem 3.1. We argue by contradiction. Suppose for each positive integer $k$, there exists a positive integer $d_{k}$ such that

$$
\operatorname{dim}_{\mathbb{C}} \mathcal{O}_{d_{k}}\left(M^{n}\right)>\operatorname{dim}_{\mathbb{C}} \mathcal{O}_{\left[\left(1-\frac{1}{k}\right) d_{k}\right]}\left(\mathbb{C}^{n}\right)
$$

Since the universal cover $\tilde{M}^{n}$ does not split isometrically and holomorphically as $\mathbb{C} \times M_{2}^{n-1}$, we have the estimate (3.1). It follows that $d_{k} \geq k$ and then

$$
\lim _{k \rightarrow \infty} \frac{\left[\left(1-\frac{1}{k}\right) d_{k}\right]}{d_{k}}=1
$$

Fix a point $x_{0} \in M^{n}$. By the Poincaré-Siegel argument, we know that for each positive integer $k$ there exists a function $f_{k} \in \mathcal{O}_{d_{k}}\left(M^{n}\right)$ such that

$$
\left[\left(1-\frac{1}{k}\right) d_{k}\right] \leq \operatorname{ord}_{x_{0}}\left(f_{k}\right)
$$

On the other hand, by (2.7) we always have

$$
\operatorname{ord}_{x_{0}}\left(f_{k}\right) \leq \operatorname{deg}\left(f_{k}\right)=d_{k}, \quad \text { for each positive integer } k \text {. }
$$

Thus

$$
\lim _{k \rightarrow \infty} \frac{\operatorname{ord}_{x_{0}}\left(f_{k}\right)}{\operatorname{deg}\left(f_{k}\right)}=1
$$

Let $u_{k}(x, t)$ solve the following heat equation:

$$
\left\{\begin{array}{l}
\frac{\partial}{\partial t} u_{k}=\Delta u_{k}, \quad \text { on } M^{n} \times(0,+\infty) \\
\left.u_{k}\right|_{t=0}=\log \left|f_{k}\right|^{2}, \quad \text { on } M^{n}
\end{array}\right.
$$

and let $w_{k}(x, t)=\Delta u_{k}(x, t)$. We have seen in Lemma 2.2 and (2.6), for each $k$,

$$
t w_{k}\left(x_{0}, t\right) \geq\left[\left(1-\frac{1}{k}\right) \operatorname{deg}\left(f_{k}\right)\right]
$$

and

for all $t>0$ and $x \in M^{n}$.

Set

$$
t w_{k}(x, t) \leq \operatorname{deg}\left(f_{k}\right)
$$

$$
\left(\tilde{u}_{k}\right)_{i \bar{j}}=\frac{1}{\operatorname{deg}\left(f_{k}\right)}\left(u_{k}\right)_{i \bar{j}} \quad \text { and } \quad \tilde{w}_{k}=\frac{1}{\operatorname{deg}\left(f_{k}\right)} w_{k} .
$$

Then we have from (2.3),

$$
\left\{\begin{aligned}
& \frac{\partial}{\partial t}\left(\tilde{u}_{k}\right)_{i \bar{j}}= \Delta\left(\tilde{u}_{k}\right)_{i \bar{j}}+R_{l \bar{m} i \bar{j}}\left(\tilde{u}_{k}\right)_{m \bar{l}} \\
&-\frac{1}{2}\left(R_{i \bar{l}}\left(\tilde{u}_{k}\right)_{l \bar{j}}+R_{l \bar{j}}\left(\tilde{u}_{k}\right)_{i \bar{l}}\right), \quad \text { on } M^{n} \times(0,+\infty), \\
& t \tilde{w}_{k}(x, t) \leq 1, \quad \text { on } M^{n} \times(0,+\infty), \\
& t \tilde{w}_{k}\left(x_{0}, t\right) \geq \frac{\left[\left(1-\frac{1}{k}\right) d_{k}\right]}{d_{k}} \rightarrow 1, \quad \text { as } k \rightarrow+\infty, \text { for all } t>0, \\
& g^{i \bar{j}}\left(\tilde{u}_{k}\right)_{i \bar{j}}=\tilde{w}_{k} \text { and } \quad\left(\left(\tilde{u}_{k}\right)_{i \bar{j}}\right) \text { is nonnegative definite on } M^{n} \times(0,+\infty) .
\end{aligned}\right.
$$


Since we have uniform bound of $\left(\tilde{u}_{k}\right)_{i \bar{j}}$ on every compact subset of $M^{n} \times(0,+\infty)$, the standard Bernstein trick for linear parabolic equations gives all the higher derivative uniform estimates of $\left(\tilde{u}_{k}\right)_{i \bar{j}}$ on every compact subset of $M^{n} \times(0,+\infty)$. Then there exists a subsequence of $k \rightarrow \infty$ so that

$$
\begin{gathered}
\left(\tilde{u}_{k}\right)_{i \bar{j}} \rightarrow \tilde{u}_{i \bar{j}}, \\
\tilde{w}_{k} \rightarrow \tilde{w}
\end{gathered}
$$

for some smooth nonnegative (1,1)-form $\tilde{u}_{i \bar{j}}$ and some smooth function $\tilde{w}$ in $C^{\infty}$ topology over compact sets of $M^{n} \times(0,+\infty)$, which satisfy

$$
\left\{\begin{array}{l}
\frac{\partial}{\partial t} \tilde{u}_{i \bar{j}}=\Delta \tilde{u}_{i \bar{j}}+R_{l \bar{k} i \bar{j}} \tilde{u}_{k \bar{l}}-\frac{1}{2}\left(R_{i \bar{l}} \tilde{u}_{l \bar{j}}+R_{l \bar{j}} \tilde{u}_{i \bar{l}}\right), \quad \text { on } M^{n} \times(0,+\infty), \\
t \tilde{w}(x, t) \leq 1, \quad \text { on } M^{n} \times(0,+\infty), \\
t \tilde{w}\left(x_{0}, t\right) \geq 1, \quad \text { for all } t>0, \\
g^{i \bar{j}} \tilde{u}_{i \bar{j}}=\tilde{w} \quad \text { and } \quad\left(\tilde{u}_{i \bar{j}}\right) \text { is nonnegative definite on } M^{n} \times(0,+\infty) .
\end{array}\right.
$$

In particular, we have

$$
t \tilde{w}\left(x_{0}, t\right) \equiv 1, \quad \text { for all } t>0 .
$$

Clearly we still have the Li-Yau-Hamilton inequality (2.4) as

$$
Z(V)=\tilde{w}_{t}+\nabla_{k} \tilde{w} \cdot V^{k}+\nabla_{\bar{k}} \tilde{w} \cdot V^{\bar{k}}+\tilde{u}_{i \bar{j}} V^{i} V^{\bar{j}}+\frac{\tilde{w}}{t} \geq 0
$$

on $M^{n} \times(0,+\infty)$ for any $(1,0)$ vector field $V$. Thus the same argument in the proof of the rigidity part of Theorem 1.2 works in the present situation. Therefore we conclude that the universal cover $\tilde{M}^{n}$ splits as

$$
\tilde{M}^{n}=M_{1}^{p} \times \mathbb{C}^{n-p}
$$

isometrically and holomorphically for some $p \leq n-1$, which contradicts the assumption.

A natural question is to ask if we can choose the positive constant $\epsilon \in(0,1)$ in Theorem 3.1 independent of the Kähler manifold. We now present a family of examples to show that it is impossible. In 1], Cao constructed a family of complete Kähler metrics of the form

$$
g_{i \bar{j}}^{\lambda}=\partial_{i} \partial_{\bar{j}} u_{\lambda}\left(\log |z|^{2}\right), \quad \lambda \in(1,+\infty),
$$

on $\mathbb{C}^{n}$ which are expanding Kähler-Ricci solitons, where $u_{\lambda}: \mathbb{R} \rightarrow \mathbb{R}$ is a suitable smooth convex and increasing function for each $\lambda \in(1,+\infty)$. It was also shown by Cao [1] that these Kähler metrics have positive sectional curvature everywhere on $\mathbb{C}^{n}$. It was further shown in [6] that these Kähler metrics have positive curvature in the sense of Nakano (i.e., positive curvature operator on the subspace of $(1,1)$ forms), and the geodesic distance from the origin of $\mathbb{C}^{n}$ with respect to the metric $g_{i \bar{j}}^{\lambda}$ (for each $\lambda \in(1,+\infty)$ ) is asymptotic to $\sqrt{\lambda}|z|^{\frac{1}{\lambda}}$ as $|z| \rightarrow+\infty$. Thus for each $\epsilon \in(0,1)$, we properly choose a $\lambda \in(1,+\infty)$ such that the Kähler manifold $M^{n}=$ $\left(\mathbb{C}^{n}, g_{i \bar{j}}^{\lambda}\right)$ acquires the equalities for all $d$ in the improved dimension estimate of Theorem 3.1. 


\section{Generalizations}

The main purpose of this section is to prove Theorem 1.4 in the Introduction. Let us begin with a lemma on exhausting functions which does not demand a upper bound on the curvature.

Lemma 4.1. Let $M^{n}$ be a complete noncompact Kähler manifold of complex dimension $n$ with nonnegative holomorphic bisectional curvature. Then there exists a positive constant $C(n)$ depending only on the dimension $n$ such that for any $a>0$, there is a smooth function $\varphi$ on $M^{n}$ satisfying

$$
\begin{aligned}
C(n)^{-1}\left(1+\frac{r\left(x, x_{0}\right)}{a}\right) & \leq \varphi(x) \leq C(n)\left(1+\frac{r\left(x, x_{0}\right)}{a}\right), \quad x \in M^{n}, \\
|\nabla \varphi| & \leq \frac{C(n)}{a}, \quad \text { on } M^{n}, \\
\left|\varphi_{i \bar{j}}\right| & \leq \frac{C(n)}{a^{2}}, \quad \text { on } M^{n},
\end{aligned}
$$

where $x_{0}$ is a fixed point in $M^{n}$ and $r\left(x, x_{0}\right)$ is the geodesic distance between $x$ and $x_{0}$.

Proof. Consider the following heat equation:

$$
\left\{\begin{array}{l}
\frac{\partial u}{\partial t}=\Delta u, \quad \text { on } M^{n} \times(0,+\infty) \\
\left.u\right|_{t=0}=r\left(x, x_{0}\right)+1, \quad \text { on } M^{n}
\end{array}\right.
$$

It is clear (see for example Lemma 1.2 in [19]) that the solution $u(x, t)$ exists for all $t \in(0,+\infty)$, and $u(x, t)$ can be represented by

$$
u(x, t)=\int_{M^{n}} H(x, y, t)\left(r\left(y, x_{0}\right)+1\right) d y .
$$

By the heat kernel estimate of Li-Yau [16, it is not hard (see for example Corollary 1.4 in [19]) to see

$$
C(n)^{-1}\left(1+r\left(x, x_{0}\right)\right) \leq u(x, 1) \leq C(n)\left(1+r\left(x, x_{0}\right)\right), \quad \text { for } x \in M^{n},
$$

for some positive constant $C(n)$ depending only on the dimension $n$.

From the heat equation (4.1) and the complex Lichnerowicz-Laplacian heat equation $(2.3)$, it is not hard to see

$$
\begin{gathered}
\left(\frac{\partial}{\partial t}-\Delta\right)|\nabla u|^{2}=-\left|u_{i \bar{j}}\right|^{2}-\left|u_{i j}\right|^{2}-2 R_{i \bar{j}} u^{i} u^{\bar{j}} \\
\leq-\left|u_{i \bar{j}}\right|^{2} \\
\left(\frac{\partial}{\partial t}-\Delta\right)\left|u_{i \bar{j}}\right|^{2}=-\left(\left|u_{i \bar{j}, k}\right|^{2}+\left|u_{i \bar{j}, \bar{k}}\right|^{2}\right)-R_{i \bar{i} j \bar{j}}\left(\lambda_{i}-\lambda_{j}\right)^{2} \\
\leq 0
\end{gathered}
$$

where $\lambda_{i}, i=1, \cdots, n$, are the eigenvalues of $u_{i \bar{j}}$ with respect to the metric $g_{i \bar{j}}$ and we have used the curvature condition in the above two inequalities.

The maximum principles for the solutions of the heat equation (4.1) with at most exponential growth on complete noncompact Kähler manifolds with nonnegative holomorphic bisectional curvature have been well developed by Ni-Tam in [19] (especially, Theorem 2.1 and Theorem 3.1 and their proofs in [19]). In particular, the maximum principles for $\nabla u(x, t)$ and $u_{i \bar{j}}(x, t)$ work in our present case because the solution $u(x, t)$ is at most linear growth. It was proved in [15] that 
both $|\nabla u|(x, t)$ and $\left|u_{i \bar{j}}\right|(x, t)$ are subsolutions of the heat equation. Since $|\nabla r|=1$, we have

$$
|\nabla u|(x, t) \leq 1, \quad \text { for all } x \in M^{n}, t>0 .
$$

To estimate $\left|u_{i \bar{j}}\right|(x, t)$, let $v(x, t)=t\left|u_{i \bar{j}}(x, t)\right|+|\nabla u(x, t)|^{2}$. By equation (4.3) and a direct computation, we have

$$
\left\{\begin{array}{l}
\left(\frac{\partial}{\partial t}-\Delta\right) v(x, t) \leq 1, \quad \text { on } M^{n} \times(0,+\infty), \\
\left.v\right|_{t=0} \leq 1, \quad \text { on } M^{n} .
\end{array}\right.
$$

which implies that

$$
\left|u_{i \bar{j}}\right|(x, 1) \leq 2, \quad \text { for all } x \in M^{n} .
$$

Summarizing (4.2), (4.5) and (4.6), we obtain the desired function $\varphi(x)=u(x, 1)$ for $a=1$. For general $a>0$, we consider the metric $\tilde{g}_{i \bar{j}}=\frac{1}{a^{2}} g_{i \bar{j}}$ on $M^{n}$. Obviously the holomorphic bisectional curvature is still nonnegative. By applying the result for the case $a=1$, there exists a function $\varphi_{a}$ on $\left(M^{n}, \tilde{g}_{i \bar{j}}\right)$ such that

$$
\begin{gathered}
C(n)^{-1}\left(1+\tilde{r}\left(x, x_{0}\right)\right) \leq \varphi_{a}(x) \leq C(n)\left(1+\tilde{r}\left(x, x_{0}\right)\right), \\
\left|\tilde{\nabla} \varphi_{a}\right| \tilde{g}_{i \bar{j}} \leq C(n), \\
\left|\left(\varphi_{a}\right)_{i \bar{j}}\right|_{\tilde{g}_{i \bar{j}}} \leq C(n), \quad \text { on } M^{n} .
\end{gathered}
$$

Since $\tilde{r}\left(x, x_{0}\right)=\frac{1}{a} r\left(x, x_{0}\right),\left|\tilde{\nabla} \varphi_{a}\right|_{\tilde{g}_{i \bar{j}}}=a\left|\nabla \varphi_{a}\right|_{g_{i \bar{j}}}$ and $\left|\left(\varphi_{a}\right)_{i \bar{j}}\right|_{\tilde{g}_{i \bar{j}}}=a^{2}\left|\left(\varphi_{a}\right)_{i \bar{j}}\right|_{g_{i \bar{j}}}$, the function $\varphi_{a}(x)$ fulfills all the requirements of the lemma. Therefore the proof of Lemma 4.1 is completed.

It is clear that for the exhausting function $\varphi$ in Lemma 4.1, there holds

$$
B\left(x_{0}, \frac{a}{C(n)}\right) \subset\{\varphi \leq C(n)+1\} \subset B\left(x_{0}, C(n)(C(n)+1) a\right) .
$$

We are now ready to prove Theorem 1.4.

Proof of Theorem 1.4. Let $M^{n}$ be a complete noncompact Kähler manifold of complex dimension $n$ with nonnegative holomorphic bisectional curvature satisfying

$$
\operatorname{Vol}\left(B\left(x_{0}, r\right)\right) \leq C(1+r)^{2 k}, \quad \text { for all } r \geq 0,
$$

for some $1 \leq k \leq n$. In view of Theorem 1.2 , we may assume $k<n$. Let $\mathcal{P}$ denote the set of all smooth plurisubharmonic functions of at most logarithmic growth. For the fixed point $x_{0} \in M^{n}$, define a number

$$
r_{x_{0}}=\max \left\{r \mid h \in \mathcal{P},\left(h_{i \bar{j}}\left(x_{0}\right)\right) \text { has } \operatorname{rank} r\right\} .
$$

Obviously $0 \leq r_{x_{0}} \leq n$. We first claim

$$
r_{x_{0}} \leq[k]<n .
$$

Suppose not; then there exists a plurisubharmonic function $h \in \mathcal{P}$ such that $\left(h_{i \bar{j}}\left(x_{0}\right)\right)$ has rank at least $[k]+1$. Obviously by adding a constant, we may assume $h\left(x_{0}\right) \geq 2$. We modify the function $h$ as follows. First we set

$$
\tilde{h}=\max \{h, 1\} \geq 1
$$

which is a continuous plurisubharmonic function of logarithmic growth. Next we evolve the function $\tilde{h}$ by the heat equation to get a solution $h(x, t)$ on $M^{n} \times$ $(0,+\infty)$. By applying the results of Ni-Tam [19] (see Theorem 3.1, Theorem 2.1 and Corollary 1.4 in [19]) we know that for fixed $t=1$, the function $h(x, 1)$ is a 
smooth plurisubharmonic function on $M^{n},\left(h_{i \bar{j}}\left(x_{0}, 1\right)\right)$ also has rank $r_{x_{0}} \geq[k]+1$, and $h(x, 1)$ is still of logarithmic growth and satisfies

$$
1 \leq h(x, 1) \leq C \log \left(2+r\left(x, x_{0}\right)\right), \quad \text { on } M^{n},
$$

for some positive constant $C$. In the following we use this new function $h(x, 1)$ to replace the original one and still denote it by $h$.

Since $\left(h_{i \bar{j}}\left(x_{0}\right)\right)$ has rank at least $[k]+1$, we have

$$
(\sqrt{-1} \partial \bar{\partial} h)^{[k]+1} \wedge \omega^{n-([k]+1)}>0, \quad \text { in a small neighborhood of } x_{0} \in M^{n},
$$

where $\omega=\sqrt{-1} g_{i \bar{j}} d z^{i} \wedge d \bar{z}^{j}$ is the Kähler form associated to the Kähler metric $g_{i \bar{j}}$. For any $a>2$ let $\varphi$ be the exhausting function obtained in Lemma 4.1. Then there exists a positive constant $\delta>0$ independent of $a$ such that

$$
0<\delta \leq \int_{\{\varphi \leq C(n)+1\}}\left(1-\frac{\varphi}{(C(n)+1)}\right)^{2([k]+1)}(\sqrt{-1} \partial \bar{\partial} h)^{[k]+1} \wedge \omega^{n-([k]+1)} .
$$

On the other hand, by Lemma 4.1, (4.7) and (4.10), we get from integrating by parts

$$
\begin{aligned}
& \int_{\{\varphi \leq C(n)+1\}}\left(1-\frac{\varphi}{(C(n)+1)}\right)^{2([k]+1)}(\sqrt{-1} \partial \bar{\partial} h)^{[k]+1} \wedge \omega^{n-([k]+1)} \\
\leq & \frac{\tilde{C}(n)}{a^{2}} \int_{\{\varphi \leq C(n)+1\}}\left(1-\frac{\varphi}{(C(n)+1)}\right)^{2[k]} h(\sqrt{-1} \partial \bar{\partial} h)^{[k]} \wedge \omega^{n-[k]} \\
\leq & \frac{C \tilde{C}(n) \log a}{a^{2}} \int_{\{\varphi \leq C(n)+1\}}\left(1-\frac{\varphi}{(C(n)+1)}\right)^{2[k]}(\sqrt{-1} \partial \bar{\partial} h)^{[k]} \wedge \omega^{n-[k]} \\
\leq & \leq \frac{C^{([k]+1)} \tilde{C}(n)(\log a)^{([k]+1)}}{a^{2([k]+1)}} \int_{\{\varphi \leq C(n)+1\}} \omega^{n} \\
\leq & \frac{C^{([k]+1)} \tilde{C}(n)(\log a)^{([k]+1)}}{a^{2([k]+1)}} \operatorname{Vol}\left(B\left(x_{0}, C(n)(C(n)+1) a\right)\right),
\end{aligned}
$$

where $\tilde{C}(n)$ denotes various positive constants depending only on the dimension $n$.

The combination of (4.11) and (4.12) gives

$$
\operatorname{Vol}\left(B\left(x_{0}, a\right)\right) \geq C \frac{a^{2([k]+1)}}{(\log a)^{([k]+1)}}, \quad \forall a \geq 2,
$$

for some positive constant $C$ independent of $a$. Since $[k]+1>k$, we arrive at a contradiction with the volume growth assumption (4.8). Thus we have proved the claim of (4.9).

Let $h$ be a smooth plurisubharmonic function of logarithmic growth such that $\left(h_{i \bar{j}}\left(x_{0}\right)\right)$ has the maximal rank $r_{x_{0}}$. Evolve the function $h$ by the heat equation

$$
\begin{cases}\frac{\partial u}{\partial t}=\Delta u, & \text { on } M^{n} \times(0,+\infty), \\ \left.u\right|_{t=0}=h, & \text { on } M^{n} .\end{cases}
$$

Clearly the smooth solution $u(x, t)$ exists for all $t \in(0,+\infty)$. Also, by applying the results of Ni-Tam [19] again, we know that for each fixed $t>0$, the function 
$u(\cdot, t)$ is still a smooth plurisubharmonic function of logarithmic growth on $M^{n}$ and $\left(u_{i \bar{j}}\left(x_{0}, t\right)\right)$ still has the maximal rank $r_{x_{0}}$. Then as before by using Corollary 2.1 in [19], we know that the kernel space $K(x, t)$ of $\left(u_{i \bar{j}}(x, t)\right)$ is a distribution which is invariant under parallel translations and then $\left(u_{i \bar{j}}(x, t)\right)$ has rank $r_{x_{0}}$ everywhere. Moreover the universal cover $\tilde{M}^{n}$ splits isometrically and holomorphically as

$$
\tilde{M}^{n}=M_{1}^{n-r_{x_{0}}} \times M_{2}^{r_{x_{0}}},
$$

where $K$ corresponds the tangent bundle of $M_{1}^{n-r_{x_{0}}}$ and $\left(u_{i \bar{j}}(x, t)\right)>0$ on $M_{2}^{r_{x_{0}}} \times$ $(0,+\infty)$. Both $M_{1}^{n-r_{x_{0}}}$ and $M_{2}^{r_{x_{0}}}$ are complete Kähler manifolds with nonnegative holomorphic bisectional curvature. The estimate (4.9) shows that the complex dimension of the kernel $K$ is at least $n-[k] \geq 1$.

Clearly for any $f \in \mathcal{O}_{d}\left(M^{n}\right)$, we can lift $f$ as a function in $\mathcal{O}_{d}\left(\tilde{M}^{n}\right)$, still denoted by $f$. In the following we will show that such $f$ must descend to a function of $\mathcal{O}_{d}\left(M_{2}^{r_{x_{0}}}\right)$.

For any nonconstant $f \in \mathcal{O}_{d}\left(M^{n}\right)$, let $u_{f}(x, t)$ solve the heat equation

$$
\left\{\begin{array}{l}
\frac{\partial}{\partial t} u_{f}=\Delta u_{f}, \quad \text { on } M^{n} \times(0,+\infty), \\
\left.u_{f}\right|_{t=0}=\log \left(|f|^{2}+1\right), \quad \text { on } M^{n} .
\end{array}\right.
$$

We see as before by applying the results of Ni-Tam 19 that the solution $u_{f}$ is smooth plurisubharmonic with logarithmic growth on $M^{n}$ for each $t \in(0,+\infty)$ and there exists $\tilde{t}>0$ such that for any $0<t<\tilde{t}$, the kernel space $K_{f}(x, t)$ is a distribution which is invariant under parallel translations, and the universal cover $\tilde{M}^{n}$ splits isometrically and holomorphically as

$$
\tilde{M}^{n}=\left(M_{f}\right)_{1}^{n-q} \times\left(M_{f}\right)_{2}^{q}
$$

with $\left.\left(\left(u_{f}\right)_{i \bar{j}}\right)\right|_{\left(M_{f}\right)_{1}^{n-q}} \equiv 0$ and $\left.\left(\left(u_{f}\right)_{i \bar{j}}\right)\right|_{\left(M_{f}\right)_{2}^{q}}>0$. Here we have lifted the function $u_{f}$ to the universal cover and still denoted it by $u_{f}$. Recall that $\left(u_{i \bar{j}}(x, t)\right)$ has maximal rank $r_{x_{0}}$ everywhere. It follows that the orthogonal complement $K_{f}^{\perp}\left(x_{0}, t\right)$ of $K_{f}\left(x_{0}, t\right)$ is contained in the orthogonal complement $K^{\perp}\left(x_{0}, t\right)$ of $K\left(x_{0}, t\right)$, otherwise $\left(u+u_{f}\right)_{i \bar{j}}\left(x_{0}, t\right)(t \in(0, \tilde{t}))$ would have rank $\geq r_{x_{0}}+1$, which contradicts with the definition of $r_{x_{0}}$. Then by the parallel translation invariance of $K_{f}^{\perp}$ and $K^{\perp}$ we have

and

$$
K_{f}^{\perp} \subset K^{\perp}
$$

Let us write $x=\left(x_{1}, x_{2}\right) \in M^{n}=\left(M_{f}\right)_{1}^{n-q} \times\left(M_{f}\right)_{2}^{q}$ with $x \in\left(M_{f}\right)_{1}^{n-q}$ and $x_{2} \in\left(M_{f}\right)_{2}^{q}$. Since $\left.\left(\left(u_{f}\right)_{i \bar{j}}\right)\right|_{\left(M_{f}\right)_{1}^{n-q}} \equiv 0$, we know that for each fixed $t \in(0, \tilde{t})$ and arbitrarily fixed $x_{2} \in\left(M_{f}\right)_{2}^{q}$, the function $u_{f}\left(\cdot, x_{2}, t\right)$ is a harmonic function of logarithmic growth. We then conclude from Cheng-Yau 4 that for each fixed $t \in(0, \tilde{t}), u_{f}$ is only a function of the second variable $x_{2} \in\left(M_{f}\right)_{2}^{q}$. This implies from Yau's Liouville theorem 22] that $f$ is only a function of variable $x_{2} \in\left(M_{f}\right)_{2}^{q}$. Thus by combining with (4.14), we have

$$
\mathcal{O}_{d}\left(M^{n}\right) \subset \mathcal{O}_{d}\left(M_{2}^{r_{x_{0}}}\right),
$$

in particular,

$$
\operatorname{dim}_{\mathbb{C}} \mathcal{O}_{d}\left(M^{n}\right) \leq \operatorname{dim}_{\mathbb{C}} \mathcal{O}_{d}\left(M_{2}^{r_{x_{0}}}\right)
$$


Therefore by using Theorem 1.2 and (4.9), we deduce that

$$
\operatorname{dim}_{\mathbb{C}} \mathcal{O}_{d}\left(M^{n}\right) \leq \operatorname{dim}_{\mathbb{C}} \mathcal{O}_{[d]}\left(\mathbb{C}^{[k]}\right), \quad \text { for each } d>0 .
$$

We next discuss the rigidity part of Theorem 1.4. Suppose there exists a positive integer $d$ such that

$$
\operatorname{dim}_{\mathbb{C}} \mathcal{O}_{d}\left(M^{n}\right)=\operatorname{dim}_{\mathbb{C}} \mathcal{O}_{d}\left(\mathbb{C}^{[k]}\right) .
$$

From (4.16) and (4.17) we know that

$$
r_{x_{0}}=[k]
$$

and

$$
\operatorname{dim}_{\mathbb{C}} \mathcal{O}_{d}\left(M_{2}^{r_{x_{0}}}\right)=\operatorname{dim}_{\mathbb{C}} \mathcal{O}_{d}\left(\mathbb{C}^{[k]}\right)
$$

Thus by Theorem 1.2 we deduce that $M_{2}^{r_{x_{0}}}$ is holomorphically isometric to $\mathbb{C}^{[k]}$. So

$$
\tilde{M}^{n}=M_{1}^{n-[k]} \times \mathbb{C}^{[k]}
$$

isometrically and holomorphically. Also, by combining with the inclusion (4.15) we have

$$
\mathcal{O}_{d}\left(M^{n}\right)=\mathcal{O}_{d}\left(\tilde{M}^{n}\right)=\mathcal{O}_{d}\left(\mathbb{C}^{[k]}\right) .
$$

This says that every function in $\mathcal{O}_{d}\left(\mathbb{C}^{[k]}\right)\left(\subset \mathcal{O}_{d}\left(\tilde{M}^{n}\right)\right)$ is $\pi_{1}\left(M^{n}\right)$-invariant. In particular, the coordinate functions $z^{1}, \cdots, z^{[k]}$ of $\mathbb{C}^{[k]}$ (regarded as holomorphic functions of $\left.\tilde{M}^{n}=M_{1}^{n-[k]} \times \mathbb{C}^{[k]}\right)$ are $\pi_{1}\left(M^{n}\right)$-invariant.

Let $\sigma \in \pi_{1}\left(M^{n}\right)$ be any deck transformation of $\tilde{M}^{n}$ and let $x_{2}^{0}=\left(z_{0}^{1}, \cdots, z_{0}^{[k]}\right)$ be any fixed point in $\mathbb{C}^{[k]}$. By the $\pi_{1}\left(M^{n}\right)$-invariance of $z^{1}, \cdots, z^{[k]}$, we have

$$
\sigma\left(\bigcap_{i=1}^{[k]}\left[z^{i}=z_{0}^{i}\right]\right)=\bigcap_{i=1}^{[k]}\left[z^{i}=z_{0}^{i}\right],
$$

i.e.,

$$
\sigma\left(M_{1}^{n-[k]} \times\left\{x_{2}^{0}\right\}\right)=M_{1}^{n-[k]} \times\left\{x_{2}^{0}\right\} .
$$

Then for each fixed $x_{2}^{0} \in \mathbb{C}^{[k]}$, the deck transformation induces a transformation, denoted by $\sigma_{x_{2}^{0}}$, on $M_{1}^{n-[k]}$. Let $x_{1} \in M_{1}^{n-[k]}$, and let $x_{2}, x_{2}^{\prime} \in \mathbb{C}^{[k]}$ be arbitrary. Then the reduced transformation $\sigma_{x_{2}}$ and $\sigma_{x_{2}^{\prime}}$ are given by

$$
\sigma\left(x_{1}, x_{2}\right)=\left(\sigma_{x_{2}}\left(x_{1}\right), x_{2}\right)
$$

and

$$
\sigma\left(x_{1}, x_{2}^{\prime}\right)=\left(\sigma_{x_{2}^{\prime}}\left(x_{1}\right), x_{2}^{\prime}\right) .
$$

Since the deck transformation $\sigma$ is isometric on $\tilde{M}^{n}=M_{1}^{n-[k]} \times \mathbb{C}^{[k]}$, we deduce that

$$
\sigma_{x_{2}}\left(x_{1}\right)=\sigma_{x_{2}^{\prime}}\left(x_{1}\right), \quad \text { for all } x_{1} \in M_{1}^{n-[k]} .
$$

This implies that the action $\pi_{1}\left(M^{n}\right)$ on $\tilde{M}^{n}=M_{1}^{n-[k]} \times \mathbb{C}^{[k]}$ splits and acts trivially on the second factor $\mathbb{C}^{[k]}$. Hence

$$
M^{n}=\left(M_{1}^{n-[k]} / \pi_{1}\left(M^{n}\right)\right) \times \mathbb{C}^{[k]}
$$

isometrically and holomorphically. Clearly the dimension estimate (4.17) implies that the first factor $\left(M_{1}^{n-[k]} / \pi_{1}\left(M^{n}\right)\right)$ cannot carry any nonconstant holomorphic function of polynomial growth.

Therefore we have completed the proof of Theorem 1.4. 
Finally it is not hard to see that the combination of the arguments of Section 3 and the above proof of Theorem 1.4 gives the following improved dimension estimate.

Proposition 4.2. Let $M^{n}$ be a complete noncompact Kähler manifold of complex dimension $n$ with nonnegative holomorphic bisectional curvature. Assume that its Ricci curvature is positive at least at one point in $M^{n}$ and suppose there exist a point $x_{0} \in M$ and positive constants $1 \leq k \leq n$ and $C>0$ such that

$$
\operatorname{Vol}\left(B\left(x_{0}, r\right)\right) \leq C(1+r)^{2 k}, \quad \text { for all } r \geq 0 .
$$

Then there exists a positive constant $\epsilon \in(0,1)$ such that

$$
\operatorname{dim}_{\mathbb{C}} \mathcal{O}_{d}\left(M^{n}\right) \leq \operatorname{dim}_{\mathbb{C}} \mathcal{O}_{[(1-\epsilon) d]}\left(\mathbb{C}^{[k]}\right),
$$

for all positive integers $d$.

\section{REFERENCES}

1. Cao, H. D., Limits of Solutions to the Kähler-Ricci flow, J. Differential Geom. 45 (1997), 257-272. MR 1449972 (99g:53042)

2. Cheeger, J., Colding, T. H. and Minicozzi, W. P., Linear growth harmonic functions on complete manifolds with nonnegative Ricci curvature, Geom. Funct. Anal. 5 (1995), no. 6, 948-954. MR.1361516 (96j:53038)

3. Colding, T. H. and Minicozzi, W. P., Harmonic functions on manifolds, Ann. of Math. (2) 146 (1997), no. 3, 725-747. MR1491451 (98m:53052)

4. Cheng, S. Y. and Yau, S. T., Differential equations on Riemannian manifolds and their geometric applications, Comm. Pure Appl. Math. 28 (1975), 333-354. MR0385749 (52:6608)

5. Chen, B. L. and Zhu, X. P., Complete Riemannian manifolds with pointwise pinched curvature, Invent. Math. 140 (2000), 423-452. MR.1757002 (2001g:53118)

6. Chen, B. L. and Zhu, X. P., Volume growth and curvature decay of positively curved Kähler manifolds, Preprint, arXiv: math.DG/0211374.

7. Demailly, J. P., $L^{2}$ vanishing theorems for positive line bundles and adjunction theory, Transcendental Methods in Algebraic Geometry, CIME, Cetrro, 1994, Lecture Notes in Math. 1646, Springer-Verlag, 1996. MR1603616 (99k:32051)

8. Donnelly, H., Harmonic functions on manifolds of nonnegative Ricci curvature, Internat. Math. Res. Notices, 2001, no. 8, 429-434. MR.1827086 (2002k:53062)

9. Hamilton, R., Eternal solutions to the Ricci flow, J. Differential Geom. 38 (1993), 1-11. MR.1231700 (94g:58043)

10. Kasue, A., Harmonic functions with growth conditions on a manifold of asymptotically nonnegative curvature II, Recent Topics in Differential and Analytic Geometry, Adv. Stud. Pure Math., vol. 18, North-Holland, 1989. MR.1145260 (93e:53043)

11. Li, P., Linear growth harmonic functions on Kähler manifolds with nonnegative Ricci curvature, Math. Res. Lett. 2 (1995), 79-94. MR1312979 (95m:53057)

12. Li, P., Harmonic sections of polynomial growth, Math. Res. Lett. 4 (1997), 35-44. MR 1432808 (98i:53054)

13. Li, P., Curvature and function theory on Riemannian manifolds, Survey in Differential Geometry vol. VII, International Press, Cambridge, 2000, 71-111. MR1919432(2003g:53047)

14. Li, P. and Tam, L. F., Complete surfaces with finite total curvature, J. Differential Geom. 33 (1991), 139-168. MR.1085138 (92e:53051)

15. Li, P. and Tam, L. F., Linear growth harmonic functions on a complete manifold, J. Differential Geom. 29 (1989), 421-425. MR0982183 (90a:58202)

16. Li, P. and Yau, S. T., On the parabolic kernel of the Schrödinger operator, Acta Math. 156 (1986), 139-168. MR0834612 (87f:58156)

17. Ni. L., Monotonicity and Kähler-Ricci flow, Preprint, arXiv: math.DG/0211214.

18. Ni, L., A monotonicity formula on complete Kähler manifolds with nonnegative bisectional curvature, Priprint, arXiv: math.DG/0307275. 
19. Ni, L. and Tam, L. F., Plurisubharmonic functions and the structure of complete Kähler manifolds with nonnegative curvature, J. Differential Geom. 64 (2003), no. 3, 457-524. MR2032112 (2005a:32023)

20. Siu, Y. T. and Yau, S. T., Complete Kähler manifolds with non-positive curvature of faster than quadratic decay, Ann. Math. 105 (1977), 225-264. MR0437797 (55:10719)

21. Yau, S. T., Some function-theoretic properties of complete Riemannian manifold and their applications to geometry, Indiana Univ. Math. J. 25 (1976), no. 7, 659-670. MR0417452 $(54: 5502)$

22. Yau, S. T., Harmonic functions on complete Riemannian manifolds, Comm. Pure Appl. Math. 28 (1975), 201-228. MR0431040 (55:4042)

23. Yau, S. T., Open problems in geometry, Lectures on Differential Geometry, by Schoen and Yau, International Press (1994), 365-404.

Department of Mathematics, Zhongshan University, Guangzhou, 510275, People's RePUBLIC OF CHINA

Department of Mathematics, Zhongshan University, Guangzhou, 510275, People's RePUBLIC OF CHINA

Department of Mathematics, Zhongshan University, Guangzhou, 510275, People's RePUBLIC OF CHINA

Department of Mathematics, Zhongshan University, Guangzhou, 510275, People's RePUBLIC OF CHINA 